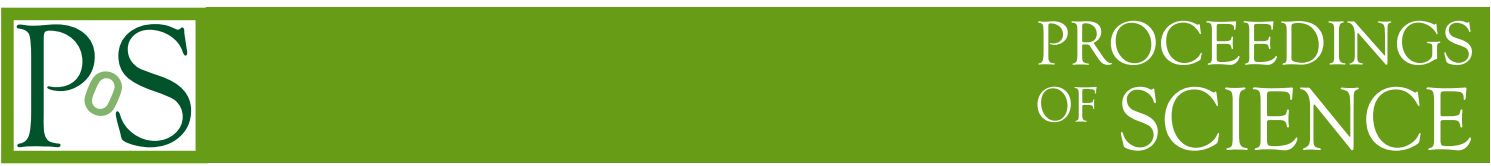

\title{
INTEGRAL and the unified model of AGN
}

\author{
Claudio Ricci* \\ ISDC Data Centre for Astrophysics \\ Geneva Observatory, University of Geneva \\ E-mail: claudio.ricci@unige.ch \\ R. Walter and T. J.-L. Courvoisier \\ ISDC Data Centre for Astrophysics \\ Geneva Observatory, University of Geneva
}

\begin{abstract}
According to the unified model, the differences observed in different classes of AGN are due to anisotropic obscuration. This implies that in the hard X-rays, at energies where the radiation can pierce the obscuring material, the average spectral characteristics of different classes of AGN should be the same. Here we present a study of the 17-250 keV INTEGRAL IBIS/ISGRI average spectra of Seyfert galaxies, aimed to constrain similarities and possible differences between different classes of AGN. The sample we consists of all the 172 Seyfert galaxies detected by INTEGRAL IBIS/ISGRI at $\mathrm{z}<0.2$ during its first 7 years of observations. Of these, 45 are Seyfert 1s, 31 Seyfert 1.5s, 67 Compton-thin Seyfert 2s, 11 Narrow Line Seyfert 1s, 11 Comptonthick Seyfert 2s, and 9 LINERs. Comparing the average hard X-ray spectra of the different classes, we found that, while the spectra of Seyfert 1s, Seyfert 1.5 and NLS1s are consistent, Comptonthin Seyfert 2 show a clear excess in the $20-60 \mathrm{keV}$ band over the spectrum of Seyfert $1 \mathrm{~s}$ and Seyfert $1.5 \mathrm{~s}$. This excess might be due to an intrinsically stronger reflection component or to a clumpy structure of the absorber in Compton-thin Seyfert 2s.
\end{abstract}

8th INTEGRAL Workshop "The Restless Gamma-ray Universe"

September 27-30 2010

Dublin Castle, Dublin, Ireland

*Speaker. 


\section{Introduction}

Active Galactic Nuclei (AGN) are thought to be powered by accretion onto a super massive black hole (1). Seyfert galaxies host AGN, and are commonly divided in Seyfert 1s and Seyfert 2s. While Seyfert 1 galaxies show both broad (FWHM $\geq 1,000 \mathrm{~km} \mathrm{~s}^{-1}$ ) and narrow (FWHM $~ 300-$ $1,000 \mathrm{~km} \mathrm{~s}^{-1}$ ) optical emission lines, Seyfert $2 \mathrm{~s}$ show only narrow lines. Seyfert $1.5 \mathrm{~s}$ show an optical spectrum intermediate between those of Seyfert 1s and Seyfert 2s. Spectropolarimetric studies of Seyfert 2s (2) showed the existence of broad emission lines also in some Seyfert 2s, which led to the formation of the unified model (UM) of AGN (3). According to this model the same engine is at work in all kind of Seyfert galaxies, and differences between Seyfert 1s and Seyfert 2s are due to the presence of an anisotropic absorber, which covers the broad line region (BLR) in Seyfert 2s. This absorber is often associated to a molecular torus. Several other lines of evidence support the $\mathrm{UM}$, like the deficit of ionizing photons in Seyfert 2 galaxies, which indicates that the ionizing source is hidden from direct view (4), and the fact that the Narrow Line Regions (NLRs) are seen with conical shapes in Seyfert 2 galaxies and halo-like shapes in Seyfert 1s (5). However, in the last years evidence of differences between Seyfert 1s and Seyfert 2s has been discovered. Seyfert 1s with significant absorption have been found (6), along with Seyfert 2s without X-ray absorption (7), and Seyferts with significantly clumpy absorbers (8). Furthermore spectropolarimetric surveys indicate that only $\sim 30-50 \%$ of the Seyfert 2 s show polarized broad lines (PBL), which might imply that not all of them harbor hidden BLRs (9),(10). The hard X-rays are particularly well suited to test the unified model, as at these energies the photons are unaffected by photoelectric absorption and it is possible to have a direct view of the X-ray source, at least up to values of the column densities $N_{\mathrm{H}} \simeq \sigma_{T}^{-1}$. For $N_{\mathrm{H}}>\sigma_{T}^{-1}$ Compton scattering starts in fact to play an important role, and only $\lesssim 50 \%$ of the incoming photons are detected. Thus, considering only Compton-thin objects, one would expect to detect consistent average spectra for different classes of AGN.

\section{Sample and data analysis}

The sample we used consists of all the 172 Seyfert galaxies detected by INTEGRAL IBIS/ISGRI (11) at $\mathrm{z}<0.2$ during its first 7 years of observations. Of these, 45 are Seyfert 1s, 31 Seyfert 1.5s, 67 Compton-thin Seyfert 2s, 11 Narrow Line Seyfert 1s (NLS1s), 11 Compton-thick (CT) Seyfert 2s and 9 LINERs. The optical classification of the sources was taken from (12). To extract the average spectra of the different AGN samples we followed the procedure adopted by (13). We created $500 \times 500$-pixels mosaic images modifying the coordinate system of each individual image, setting the coordinates of each source of the sample to an arbitrary fixed position $(\alpha=0, \delta=0)$. The geometry of the image was also modified to have consistent PSF whatever the position of the source in the field of view (FOV). These mosaic images provide a stack of all the selected IBIS/ISGRI data for each considered sample. Individual sky images for each pointing were produced in a broad energy band (i.e. 17-250 keV), and divided in 10 bins. The spectra were extracted from the mosaics using mosaic_spec. In figure 1 we show, as an example, a composite of the central part of the 17-80 keV mosaic image obtained for Seyfert 1s. 


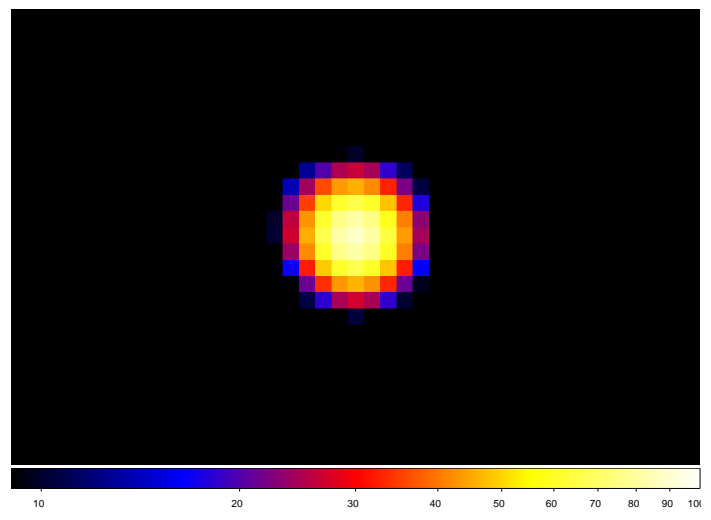

Figure 1: Composite of the central part of the $17-80 \mathrm{keV}$ mosaic image obtained for Seyfert 1s.

\section{Absorption in the $\mathrm{X}$-rays}

Both photoelectric absorption and Compton scattering must be taken into account when modeling the effects of absorption in the X-rays. The photoelectric cross section $\sigma_{p h}$ has a strong dependence on the energy, whereas Compton scattering depends on the Thomson cross section $\sigma_{\mathrm{T}}$, and it is constant with the energy, at least up to the Klein-Nishina decline. The ratio between KleinNishina and Thomson cross sections $\sigma_{\mathrm{K}-\mathrm{N}} / \sigma_{\mathrm{T}}$ starts to diverge from the unity at $E \simeq 20 \mathrm{keV}$, and it is of $\simeq 0.6$ at $200 \mathrm{keV}$. Compton processes become significant for column densities higher than $\sigma_{\mathrm{T}}^{-1}=1.5 \times 10^{24} \mathrm{~cm}^{-2}$. The cumulative effect of the two cross sections is given by:

$$
M(E)=e^{-\sigma_{p h}(E) N_{\mathrm{H}}} \times e^{-\sigma_{\mathrm{T}} N_{\mathrm{H}}} .
$$

In Fig. 2 we show the effect of absorption (considering both photoelectric absorption and Compton scattering, as reported in Eq. 3.1) on a power law model with a photon index of $\Gamma=1.95$ in the 0.1-300 keV energy range for different values of $N_{\mathrm{H}}$. In Fig. 3 we show the fraction of escaping flux for different values of ${ }_{\mathrm{H}}$ in the energy band we used. About $60 \%$ of the original emission is unabsorbed for $N_{\mathrm{H}}=7 \times 10^{23} \mathrm{~cm}^{-2}$, and we used this value as a threshold between Compton-thin and Compton-thick sources. In our final sample we considered only AGN with a value of the column density $N_{\mathrm{H}} \leq 7 \times 10^{23} \mathrm{~cm}^{-2}$. The values of the column densities were taken from soft X-ray $(E<10 \mathrm{keV})$ observations. We excluded from our sample the sources for which no value of $N_{\mathrm{H}}$ was available in the literature.

\section{Model-independent spectral analysis}

The hard X-ray spectrum of AGN can be well described by a power law continuum with an exponential cut-off, and a reflection hump peaking at $\mathrm{E} \simeq 30 \mathrm{keV}$ (14). One of the drawbacks of a model dependent analysis is the parameter degeneracy. This might not allow to well constrain several parameters at the same time. An alternative method to characterize differences and similarities between different classes of Seyfert galaxies, independently of their average flux, is through a model independent approach. This has been done by normalizing the flux of the different spectra at the first bin (i.e. 17-22 keV), and then calculating their ratio. In Fig. 4 we show the spectra and 


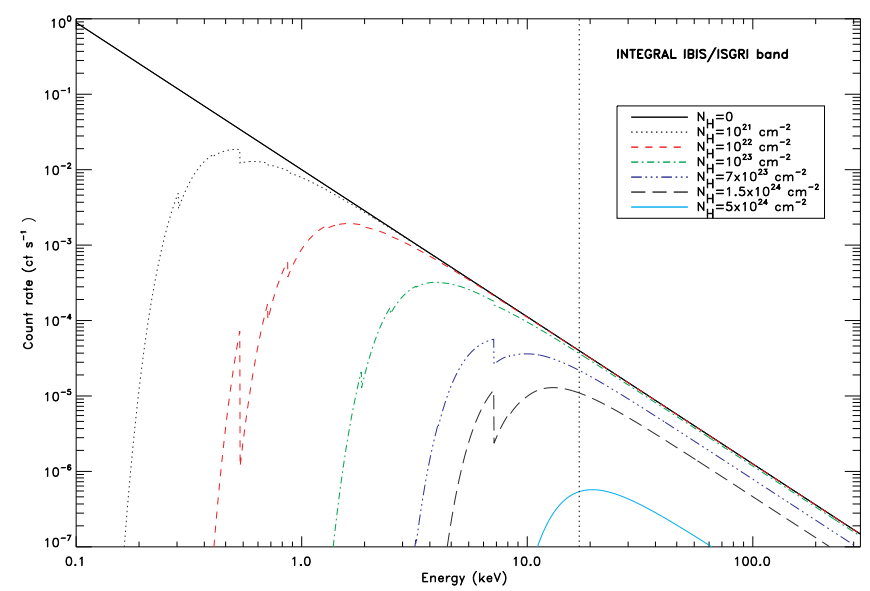

Figure 2: Effect of photoelectric absorption and Compton scattering on a power law model with a photon index of $\Gamma=1.95$ in the X-rays.

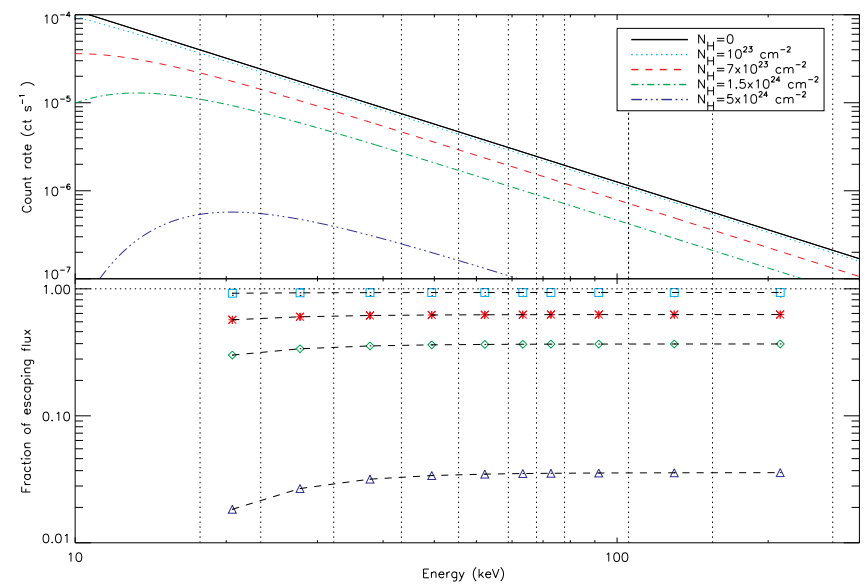

Figure 3: Effect of photoelectric absorption and Compton scattering on a power law spectrum $(\Gamma=1.95)$ in the hard X-rays. The upper panel shows the observed spectrum for different values of the hydrogen column density $N_{\mathrm{H}}$, and the lower panel shows the fraction of escaping flux in the 10 energy bins used in this work. In the lower panel squares represent the case $N_{\mathrm{H}}=10^{23} \mathrm{~cm}^{-2}$, stars $N_{\mathrm{H}}=7 \times 10^{23} \mathrm{~cm}^{-2}$, diamonds $N_{\mathrm{H}}=1.5 \times 10^{24} \mathrm{~cm}^{-2}$ and triangles $N_{\mathrm{H}}=5 \times 10^{24} \mathrm{~cm}^{-2}$.

ratios obtained comparing the average hard X-ray spectrum of Seyfert 1s to those of Seyfert 1.5s and NLS1s. As it can be seen from the figure the average spectra of both Seyfert 1.5s and NLS1s are consistent with that of Seyfert 1s. NLS1s are known to have steeper soft X-ray spectra than Seyfert 1 and Seyfert 1.5 galaxies. The fact that our sample of NLS1s is hard X-ray selected probably introduces a bias, being the objects with a flatter spectrum more easily detected than those with a steeper spectrum. (15) studying a smaller sample of NLS1s found that their hard X-ray spectrum is on average steeper than that of Seyfert 1s. The difference between our results and theirs is probably due to the lower exposures and smaller energy ranges used in their work.

Given the similarity of the spectra of Seyfert 1s and Seyfert 1.5s, we re-ran the analysis considering all the objects of the two samples, obtaining an average spectrum of Seyfert 1s and Seyfert 1.5s. 
We compared this spectrum to that of Compton-thin Seyfert 2s, obtaining a result remarkably different from what we had for the other classes. As it can be seen from Fig. 5, the average hard $\mathrm{X}$-ray spectrum of Compton-thin Seyfert $2 \mathrm{~s}$ is significantly harder in the 22-60 keV band, showing a bump which might be linked to a stronger reflection hump. At energies higher than $60 \mathrm{keV}$ the ratio of the two spectra is consistent with the unity, which implies that, although showing a stronger reflection component, Compton-thin Sy 2s have the same continuum of Sy 1s and Sy 1.5s.
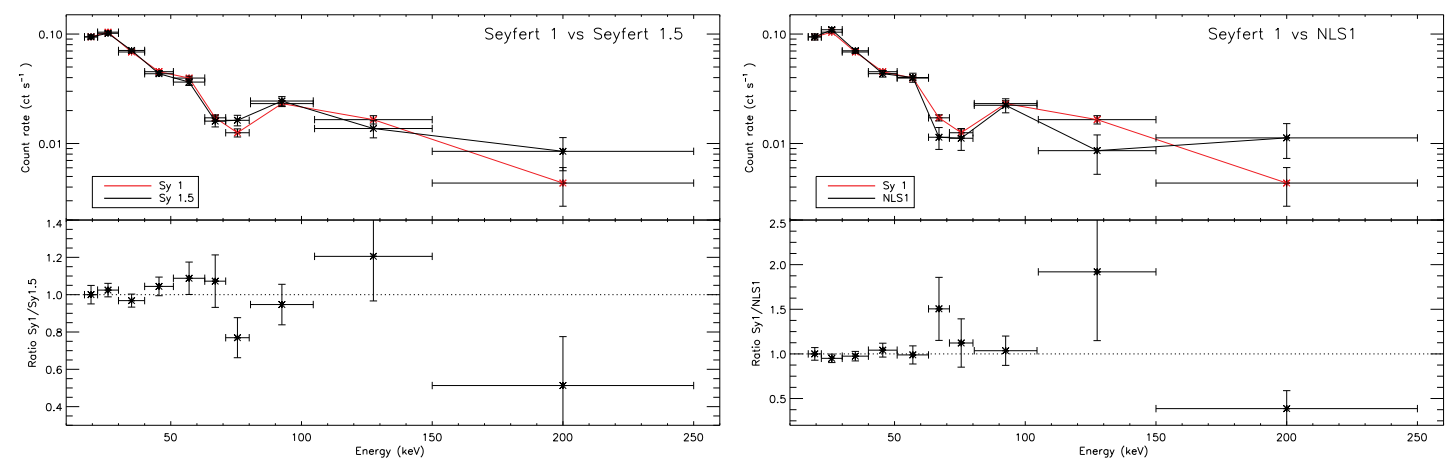

Figure 4: Spectra (upper panels) and ratios (lower panels) between the normalized spectra of Seyfert 1s and Seyfert 1.5s (left), Seyfert 1s and NLS1s (right).

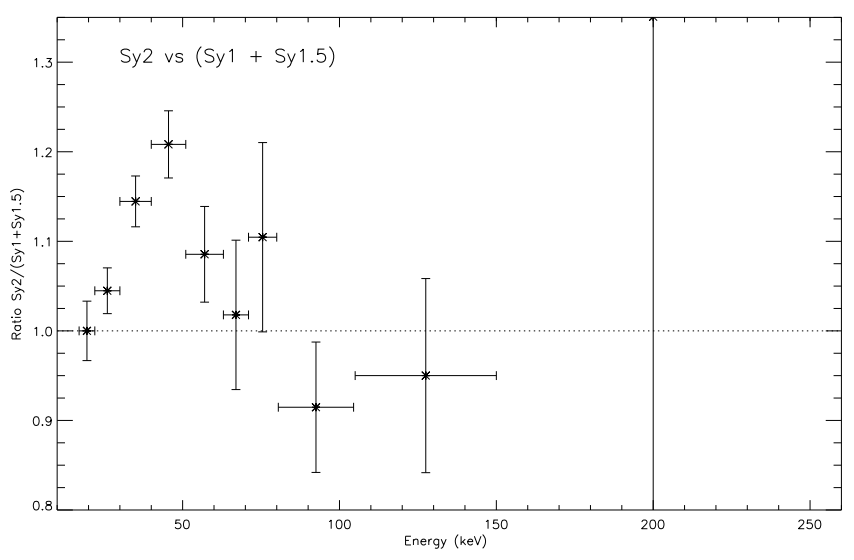

Figure 5: Ratio between the spectra of Compton-thin Seyfert 2s and the one of Seyfert 1s and Seyfert 1.5s.

\section{The unified model of AGN}

The continuum emission of different classes of Seyfert galaxies has on average the same characteristics, which confirms the idea that different classes of objects have the same engine, as predicted by the zero-th order unified model of AGN. The stronger reflection observed in the spectrum of Compton-thin Seyfert 2s cannot be explained in terms of absorption, as the chosen threshold of $N_{\mathrm{H}}$ should guarantee a transmission dominated spectrum. Moreover considering that the average column density of our sample of Seyfert $2 \mathrm{~s}$ is $N_{\mathrm{H}}=1.5 \times 10^{23} \mathrm{~cm}^{-2}$, one would expect only $\simeq 10 \%$ of the flux to be Compton scattered. The excess in the $20-60 \mathrm{keV}$ band might be related either to 
an intrinsic stronger reflection component of Seyfert $2 \mathrm{~s}$, or to the presence of clumps in the torus, as predicted by (16).

\section{Conclusions}

We presented the study of the average INTEGRAL IBIS/ISGRI hard X-ray spectra of different classes of Seyfert galaxies. We found that, as predicted by the UM, the average spectra of Seyfert 1s and Seyfert 1.5s are consistent in the $17-250 \mathrm{keV}$ band. NLS1s also show a spectrum similar to that of Seyfert 1s and Seyfert 1.5s, probably due to the hard X-ray selected nature of our sample. The average emission of Compton-thin Sy $2 \mathrm{~s}$ is harder than that of Sy $1 \mathrm{~s}$ and Sy $1.5 \mathrm{~s}$ in the 20-60 $\mathrm{keV}$ energy band, which might be due either to an intrinsically stronger Compton reflection or to a clumpy torus. The UM is confirmed on the zero-th order, although an homogenous anisotropic absorber alone cannot easily explain the differences between the spectrum of Seyfert $2 \mathrm{~s}$ and that of Seyfert $1 \mathrm{~s}$ and Seyfert $1.5 \mathrm{~s}$.

\section{References}

[1] Rees, M. J. 1984, ARA\&A 22, 471

[2] Miller, J. S., \& Antonucci, R. R. J. 1983, ApJ 271, L7

[3] Antonucci, R. R. J., \& Miller, J. S. 1985, ApJ 297, 621

[4] Schmitt, H. R., \& Kinney, A. L. 1996, ApJ 463, 498

[5] Pogge, R. W., 1988, ApJ 328, 519

[6] Cappi M., Panessa, F., Bassani, L. et al., 2006, A\&A, 446, 459

[7] Bianchi, S., Corral, A., Panessa, F., et al. 2008, MNRAS, 385, 195

[8] Ricci, C., Beckmann, V., Audard, M., et al. 2010, A\&A, 518, 47

[9] Tran, H. D. 2001, ApJ 554, L19

[10] Tran, H. D. 2003, ApJ 583, 632

[11] Ubertini, P., Lebrun, F., Di Cocco, G., 2003, A\&A, 411, 131

[12] Veron-Cetty, M.-P., Veron, P. 2010, A\&A, 518, 10

[13] Walter, R. \& Cabral, N., 2009 A\&A 497, 97

[14] Magdziarz, P., \& Zdziarski, A. A. 1995, MNRAS 273, 873

[15] Malizia, A., Bassani, L., Bird, A. J., et al. 2008, MNRAS 389, 1360

[16] Elitzur, M. \& Shlosman, I. 2006, ApJ 648L, 101 